\title{
New coordinates for a simpler canonical derivation of the Hawking effect
}

\author{
Golam Mortuza Hossain $^{\mathrm{a}}{ }^{\mathbb{D}}$, Chiranjeeb Singha $^{\mathrm{b}}{ }^{(\mathbb{D}}$ \\ Department of Physical Sciences, Indian Institute of Science Education and Research Kolkata, Mohanpur 741 246, West Bengal, India
}

Received: 30 August 2019 / Accepted: 18 January 2020 / Published online: 1 February 2020

(C) The Author(s) 2020

\begin{abstract}
In order to achieve a Hamiltonian-based canonical derivation of the Hawking effect, one usually faces multiple hurdles. Firstly, the spacetime foliation using Schwarzschild time does not lead to hyper-surfaces which are always spacelike. Secondly, the null coordinates which are frequently used in covariant approach, do not lead to a true matter Hamiltonian. Recently, an exact canonical derivation was presented using the so-called near-null coordinates. However, there too one faces the difficulty of having to deal with non-vanishing matter diffeomorphism generator as the spatial decomposition involves a non-zero shift vector. Here we introduce a new set of coordinates which allows one to perform an exact canonical derivation of Hawking effect without having to deal with matter diffeomorphism generator.
\end{abstract}

\section{Introduction}

An asymptotic future observer perceives thermal emission in a black hole spacetime when one considers quantum fields in such classical geometry. This phenomenon is known as the Hawking effect [1]. Usually, a very large number of microstates are needed to understand thermal emission from a body. However, a classical black hole can be described by only few parameters in Einstein's general theory of relativity [2-5]. So one expects that the study of Hawking effect in principle might allow one to understand the possible, yet unknown, quantum theory of gravity and significant efforts have been made to understand the Hawking effect in many different ways [6-34].

In the canonical approaches to quantum gravity, one decomposes the spacetime into spatial hyper-surfaces labeled by a suitable time parameter. Consequently, in order to explore the techniques that are often employed in such

\footnotetext{
a e-mail: ghossain@iiserkol.ac.in

be-mail: cs12ip026@iiserkol.ac.in
}

canonical quantization framework, it is desirable to have a Hamiltonian-based canonical derivation of the Hawking effect. In such an approach, however one faces multiple hurdles. Firstly, the hyper-surfaces for fixed Schwarzschild time are not always spacelike [35-37] and consequently Hamiltonian dynamics is not well-posed in such coordinates. Secondly, in the standard derivation of the Hawking effect one needs to find the relation between the ingoing and outgoing massless field modes as seen by two asymptotic observers at the past and the future null infinity respectively [1]. These field modes follow null trajectory and are conveniently described using null coordinates. However, null coordinates do not lead to a true matter Hamiltonian that can describe the dynamics of these modes.

In order to overcome these difficulties, recently a set of near-null coordinates is introduced in [38] which allows one to perform an exact canonical derivation of the Hawking effect. Firstly, these near-null coordinates lead to a non-trivial matter Hamiltonian which describes the dynamics of the field modes. Secondly, these coordinates being structurally closer to the null coordinates, allow one to follow similar methods which are employed for null coordinates. Nevertheless, the usage of these near-null coordinates leads to the off-diagonal terms in the spacetime metric. The corresponding spacetime decomposition involves both the lapse function as well as a non-vanishing shift vector. Consequently, the dynamics of field modes depends not just on matter Hamiltonian but also on the matter diffeomorphism generator.

This article is organized as follows. In the Sect. 2, we review the key aspects of a Schwarzschild black hole spacetime. Then we discuss the difficulties that one faces while using Schwarzschild time for space-time foliation. Subsequently, we introduce a new set of coordinates which allows an exact canonical derivation of the Hawking effect. The spacetime decomposition into spatial hyper-surfaces using these coordinates does not involve any shift vector. There- 
fore, the usage of these coordinates leads to a much simpler Hamiltonian-based derivation of the Hawking effect.

\section{Schwarzschild spacetime}

Let us consider a Schwarzschild spacetime which is formed at some finite past, possibly due to the collapse of a matter shell whose exact dynamics however is not important for understanding the Hawking effect. The invariant distance element in the Schwarzschild spacetime is given by

$d s^{2}=-f(r) d t^{2}+f(r)^{-1} d r^{2}+r^{2} d \theta^{2}+r^{2} \sin ^{2} \theta d \phi^{2}$,

where $f(r)=\left(1-r_{s} / r\right)$ and $r_{s}=2 G M$ is the Schwarzschild radius. Throughout the paper, we use natural units where $c=\hbar=1$. It is well-known that the Hawking effect is ultimately connected with the structure of the Schwarzschild metric in the $t-r$ plane. Therefore, for simplicity now onward we consider $1+1$ dimensional Schwarzschild spacetime with the metric $g_{\mu \nu}$ along with the invariant distance

$d s^{2}=g_{\mu \nu} d x^{\mu} d x^{\nu}=-f(r) d t^{2}+f(r)^{-1} d r^{2}$.

In order to represent the Hawking quanta, here we consider a minimally coupled massless scalar field $\Phi(x)$ whose dynamics is governed by the action

$S_{\Phi}=\int d^{2} x\left[-\frac{1}{2} \sqrt{-g} g^{\mu \nu} \partial_{\mu} \Phi(x) \partial_{\nu} \Phi(x)\right]$.

We shall ignore the back-reaction of this scalar field on the spacetime metric as done also in the standard derivation of the Hawking effect [1].

\section{Canonical formulation}

It turns out that the Schwarzchild time $t$ is not a good choice of time parameter for canonical formulation as the hyper-surfaces with a fixed Schwarzschild time $t$ are not always spacelike. We may easily see it from the expression $d s_{\mid d t=0}^{2}=f(r)^{-1} d r^{2}$ where hyper-surfaces for fixed Schwarzschild time are spacelike when $r>r_{s}$ and timelike when $r<r_{s}$ [35-37]. In order to consider the spatial region only outside the horizon, usually one defines the so-called tortoise coordinate $r_{\star}$ such that $d r_{\star}=f(r)^{-1} d r$. By choosing suitable constant of integration, $r_{\star}$ can be expressed as

$r_{\star}=r+r_{s} \ln \left(r / r_{s}-1\right)$.

The domain of $r_{\star}$ being $(-\infty, \infty)$, it covers only a part of the full Schwarzschild spacetime and the corresponding metric becomes

$d s^{2}=f(r)\left[-d t^{2}+d r_{\star}^{2}\right]$, which differs from $1+1$ dimensional Minkowski metric by a conformal transformation.

\subsection{Null coordinates}

In the standard derivation [1], the Hawking effect is realized by computing the Bogoliubov transformation coefficients between the ingoing field modes that originate from the past null infinity $\left(\mathscr{I}^{-}\right)$and the outgoing field modes that arrive at the future null infinity $\left(\mathscr{I}^{+}\right)$respectively. For massless scalar field, these field modes follow null trajectories and are conveniently described using ingoing and outgoing null coordinates, defined as

$v=t+r_{\star} ; u=t-r_{\star}$.

Subsequently, using these Bogoliubov coefficients, one computes the expectation value of number density operator corresponding to an observer near future null infinity in the vacuum state corresponding to an observer near past null infinity. This expectation value turns out to be the same as the blackbody spectrum at the Hawking temperature. Therefore, these null coordinates play key roles even in the basic formulation of the Hawking effect in the covariant approach. However, these null coordinates do not lead to a true Hamiltonian for the matter field (3) that can describe the field dynamics. Consequently, these null coordinates are not suitable for performing a Hamiltonian-based canonical derivation of the Hawking effect.

\subsection{Timelike and spacelike coordinates}

In order to perform an exact canonical derivation of the Hawking effect, a set of near-null coordinates is introduced in Ref. [38]. In particular, a timelike coordinate $\tau_{-}$and a spacelike coordinate $\xi_{-}$used by an observer near the past null infinity $\mathscr{I}^{-}$, referred to as the observer $\mathbb{O}^{-}$, are given by

$\tau_{-}=t-(1-\epsilon) r_{\star} ; \quad \xi_{-}=-t-(1+\epsilon) r_{\star}$,

where the parameter $\epsilon$ is taken to be small and positive such that $\epsilon \ll 1$ which signifies the naming of these coordinates as 'near-null'. Similarly, one introduces another set of timelike coordinate $\tau_{+}$and spacelike coordinate $\xi_{+}$for an observer near the future null infinity $\mathscr{I}^{+}$. These coordinates are given by

$\tau_{+}=t+(1-\epsilon) r_{\star} ; \quad \xi_{+}=-t+(1+\epsilon) r_{\star}$,

and the corresponding observer is referred to as the observer $\mathbb{O}^{+}$. We note that the domain of the coordinates $\tau_{ \pm}$and $\xi_{ \pm}$ both are $(-\infty, \infty)$. 


\subsubsection{Domain of the parameter $\epsilon$}

The main motivation for choosing the parameter $\epsilon$ to be very small in Ref. [38] was to keep these coordinates structurally 'near' to the null coordinates so that one could employ similar methods as used for null coordinates. However, in general, any value of the parameter $\epsilon$ in the domain $0<\epsilon<2$ allows one to maintain the timelike and spacelike characteristics of the coordinates $\tau_{ \pm}$and $\xi_{ \pm}$respectively. Therefore, these coordinates can, in principle, be used for the study of the Hawking effect using canonical formulation in the entire allowed domain of $\epsilon$ which is not necessarily small. However, such coordinates would then loose their 'near-null' characteristics. We note that for both the observers $\mathbb{O}^{+}$and $\mathbb{O}^{-}$, the $1+1$ dimensional Schwarzschild metric (5) can be expressed as

$d s^{2}=\frac{f(r)}{4}\left[-\alpha d \tau_{ \pm}^{2}+\beta d \tau_{ \pm} d \xi_{ \pm}+\gamma d \xi_{ \pm}^{2}\right]$,

where $\alpha=\left(2 \epsilon+\epsilon^{2}\right), \beta=2\left(2-\epsilon^{2}\right)$ and $\gamma=\left(2 \epsilon-\epsilon^{2}\right)$. For the small values of the parameter $\epsilon$ i.e. $\epsilon \ll 1$, the parameter $\beta$ is non-vanishing. Therefore, if one foliates the spacetime into spatial hyper-surfaces by using the time variables $\tau_{ \pm}$, the presence of the off-diagonal terms in the metric leads to nonvanishing shift vector. This in turns forces one to deal with the non-vanishing matter diffeomorphism generator [38].

\subsubsection{Parameter $\epsilon=\sqrt{2}$}

However, one may notice that the off-diagonal terms in the metric (9) vanishes identically for both observers if one chooses $\epsilon=\sqrt{2}$ which implies $\beta=0$. Then the corresponding metric becomes

$d s^{2}=\frac{f(r)}{4}\left[-\alpha d \tau_{ \pm}^{2}+\gamma d \xi_{ \pm}^{2}\right] \equiv g_{\mu \nu}^{ \pm} d x^{\mu} d x^{\nu}$,

where $\alpha=2(\sqrt{2}+1)$ and $\gamma=2(\sqrt{2}-1)$. Clearly, if we use $\tau_{ \pm}$as time parameters with $\epsilon=\sqrt{2}$, then the foliation of the spacetime into spatial hyper-surfaces does not involve any shift vector.

In order to elucidate the properties of this specific choice of the foliation, we note that for constant $\tau_{-}$surfaces, $\left(d t / d r_{\star}\right)=-(\sqrt{2}-1)=-\tan (\pi / 8)$. In other words, in the $t-r_{\star}$ plane, a spatial hyper-surface with constant $\tau_{-}$makes an angle of $\pi / 8$ with the ingoing null surfaces with constant $v$. Similarly, it is easy to see that in the $t-r_{\star}$ plane, a spatial hyper-surface with constant $\tau_{+}$makes an angle of $-\pi / 8$ with the outoing null surfaces with constant $u$ (see Fig. 1).

\subsubsection{Relation between spatial coordinates $\xi_{-}$and $\xi_{+}$}

In order to perform the canonical derivation of the Hawking effect, a key task is to find the relation between the spatial coordinates $\xi_{-}$and $\xi_{+}$which are used by the two asymptotic observers. Firstly, from the Eqs. $(7,8)$, we note that

$d \xi_{-\mid \tau_{-}}=-2 d r_{\star \mid \tau_{-}}, d \xi_{+\mid \tau_{+}}=2 d r_{\star \mid \tau_{+}}$.

However, we may emphasize here that there was no black hole when the ingoing modes relevant for Hawking effect left the $\mathscr{I}^{-}$as seen by the observer $\mathbb{O}^{-}$. So one should view the coordinates $\left(\tau_{-}, \xi_{-}\right)$subject to the condition $r_{s} \rightarrow 0$
Fig. 1 a The spacelike and timelike hyper-surfaces for the observer $\mathbb{O}^{-}$are drawn with arbitrary unit using dotted red line and solid blue line respectively for $\epsilon=\sqrt{2}$. b The spacelike and timelike hyper-surfaces for the observer $\mathbb{0}^{+}$are drawn using dotted red line and solid blue line respectively for $\epsilon=\sqrt{2}$. $\mathrm{c}$ The spacelike, timelike and null hyper-surfaces are drawn using dotted red line, solid blue line and dashed black line respectively for $\epsilon=\sqrt{2}$. d The angle made by the constant $\tau_{-}$ and $\tau_{+}$hyper-surfaces in $t-r_{\star}$ plane for different values of the parameter $\epsilon$

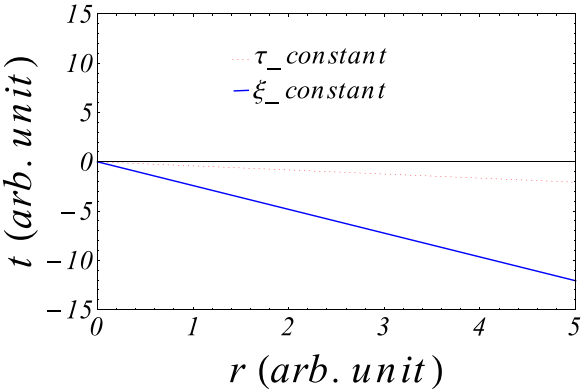

(a)

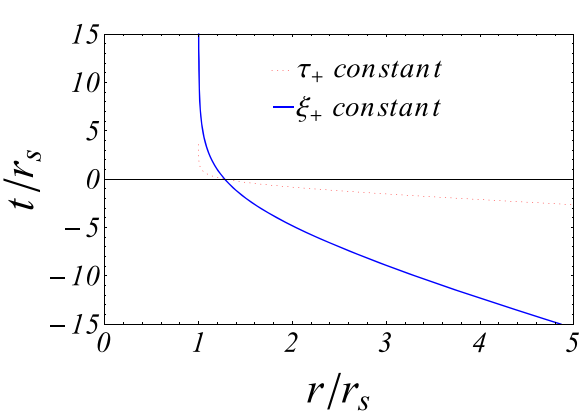

(b)

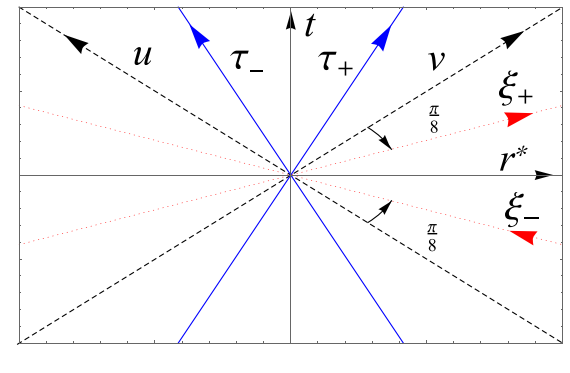

(c)

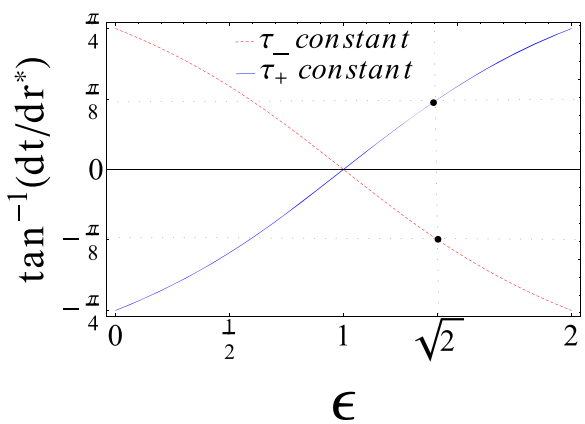

(d) 
which implies $f(r) \rightarrow 1$ and $r_{\star} \rightarrow r$. Now, using the metric (2), one can calculate the non-vanishing Christoffel symbols given by

$\Gamma_{t r}^{t}=\Gamma_{r t}^{t}=-\Gamma_{r r}^{r}=\frac{f^{\prime}(r)}{2 f(r)} ; \quad \Gamma_{t t}^{r}=\frac{1}{2} f(r) f^{\prime}(r)$.

By introducing an affine parameter $\sigma$ along the null trajectories which are defined by $d s^{2}=0$, the geodesic equations can be expressed as

$\frac{d}{d \sigma}\left(f(r) \frac{d t}{d \sigma}\right)=0, \frac{d^{2} r}{d \sigma^{2}}=0$.

The Eq. (13) admit solutions for $r$ as

$r=C \sigma+D$,

where $C, D$ are constants of integration. Given affine transformations are of the form $\sigma \rightarrow \sigma^{\prime}=C \sigma+D$, the coordinate $r$ can also be viewed as an affine parameter. We have mentioned that for the observer $\mathbb{O}^{-}$, one should view the coordinates $\left(\tau_{-}, \xi_{-}\right)$subject to the condition $r_{s} \rightarrow 0$. Now if we consider a pivotal point $\xi_{-}^{0}$ on a constant $\tau_{-}$hypersurface with $r^{0}$ being the corresponding value of the radial coordinate then the Eq. (11) implies

$\left(\xi_{-}-\xi_{-}^{0}\right)_{\mid \tau_{-}}=2\left(r^{0}-r\right)_{\mid \tau_{-}}$,

where $\left(\xi_{-}-\xi_{-}^{0}\right) \mid \tau_{-}$to be viewed as the spatial separation between any two ingoing null rays which were at the locations $\xi_{-}$and $\xi_{-}^{0}$ respectively on the spatial hyper-surfaces labelled by the time parameter $\tau_{-}$.

On the other hand, when the relevant outgoing modes for Hawking radiation arrive at $\mathscr{I}^{+}$, as seen by the observer $\mathbb{O}^{+}$, the black hole has already been formed. So if we consider a pivotal point $\xi_{+}^{0}$ on a constant $\tau_{+}$hyper-surface then using the Eqs. (4) and (11) one can express the spatial separation between two given outgoing null rays along the hyper-surface as

$\left(\xi_{+}-\xi_{+}^{0}\right)_{\mid \tau_{+}}=2\left(r-r^{0}\right)_{\mid \tau_{+}}+2 r_{s} \ln \left(1+\frac{r-r^{0}}{r^{0}-r_{s}}\right)_{\mid \tau_{+}}$.

We have already shown that the coordinate $r$ along both ingoing and outgoing null trajectories can be considered as affine parameter. Therefore, using geometric optics approximation we can relate the spatial separations of the ingoing and the outgoing modes as

$\left(r-r^{0}\right)_{\mid \tau_{+}}=C^{\prime}\left(r^{0}-r\right)_{\mid \tau_{-}}$,

where $C^{\prime}$ is some constant. Given this constant $C^{\prime}$ does not affect the final result, then for simplicity we set this value to be unity. By choosing $\xi_{-}^{0}=2\left(r^{0}-r_{s}\right){\mid \tau_{+}}_{\text {and }} \xi_{+}^{0}=$
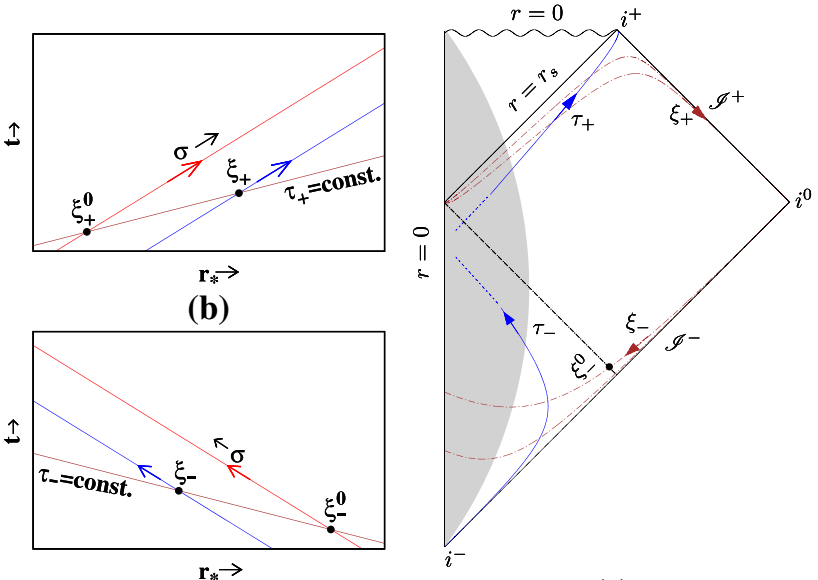

(c)

(a)

Fig. 2 a Spatial separation between two ingoing null rays along a $\tau_{-}$ constant hyper-surface. b Spatial separation between two outgoing null rays along a $\tau_{+}$constant hyper-surface. $\mathbf{c}$ The spacelike and timelike coordinates for $\epsilon=\sqrt{2}$ drawn on a Penrose diagram together with a collapsing shell of matter denoted by the shaded region

$\xi_{-}^{0}+2 r_{s} \ln \left(\xi_{-}^{0} / 2 r_{s}\right)$ in the Eq. (16), we can express it as

$\xi_{+}=\xi_{-}+2 r_{s} \ln \left(\frac{\xi_{-}}{2 r_{s}}\right)$.

In the domain where $\left|\xi_{-}\right|<<2 r_{s}$, we may approximate the relation (18) between spatial coordinates $\xi_{-}$and $\xi_{+}$as used by two asymptotic observers $\mathbb{O}^{-}$and $\mathbb{O}^{+}$respectively, as

$\xi_{-} \approx 2 r_{s} e^{\xi_{+} / 2 r_{s}}$.

The relation (19) is the key relation which ultimately leads to the Hawking effect.

\subsubsection{Scalar matter field}

We note that by using a conformally transformed spacetime metric $g_{\mu \nu}^{0}$ such that $g_{\mu \nu}^{ \pm}=\frac{1}{4} \gamma f(r) g_{\mu \nu}^{0}$, the scalar field action (3) for both the observers can be written in the form

$S_{\varphi}=\int d \tau_{ \pm} d \xi_{ \pm}\left[-\frac{1}{2} \sqrt{-g^{0}} g^{0 \mu \nu} \partial_{\mu} \varphi \partial_{\nu} \varphi\right]$

where the metric $g_{\mu \nu}^{0}$ is flat and consequently we can use the standard techniques of Fock quantization for the matter field. Using the time coordinates $\tau_{ \pm}$, we can compute the scalar matter Hamiltonian as

$H_{\varphi}^{ \pm}=\int d \xi_{ \pm} N\left[\frac{\Pi^{2}}{2 \sqrt{q}}+\frac{\sqrt{q}}{2}\left(\partial_{\xi_{ \pm}} \varphi\right)^{2}\right]$

where the lapse function $N=\sqrt{\alpha / \gamma}=(\sqrt{2}+1)$ and the determinant of the spatial metric $q=1$. The Poisson bracket between the field $\varphi$ and its conjugate momentum $\Pi$ for both 
the observers can be expressed as

$\left\{\varphi\left(\tau_{ \pm}, \xi_{ \pm}\right), \Pi\left(\tau_{ \pm}, \xi_{ \pm}^{\prime}\right)\right\}=\delta\left(\xi_{ \pm}-\xi_{ \pm}^{\prime}\right)$.

Using the equations of motion, the field momentum $\Pi$ can be expressed as

$\Pi\left(\tau_{ \pm}, \xi_{ \pm}\right)=\frac{\sqrt{q}}{N}\left(\partial_{\tau_{ \pm}} \varphi\right)$

\subsubsection{Fourier modes}

The spatial volume $V_{ \pm}=\int d \xi_{ \pm} \sqrt{q}$ is formally divergent. Therefore, to avoid dealing with explicitly divergent quantity, we choose a fiducial box with finite volume as

$V_{ \pm}=\int_{\xi_{ \pm}^{L}}^{\xi_{ \pm}^{R}} d \xi_{ \pm} \sqrt{q}=\xi_{ \pm}^{R}-\xi_{ \pm}^{L} \equiv L_{ \pm}$,

where $\xi_{ \pm}^{L}$ and $\xi_{ \pm}^{R}$ are left and right coordinate edges associated with the box. We may now define the Fourier modes for the scalar field as [39]

$\varphi\left(\tau_{ \pm}, \xi_{ \pm}\right)=\frac{1}{\sqrt{V_{ \pm}}} \sum_{k} \tilde{\phi}_{k}^{ \pm}\left(\tau_{ \pm}\right) e^{i k \xi_{ \pm}}$

$\Pi\left(\tau_{ \pm}, \xi_{ \pm}\right)=\frac{1}{\sqrt{V_{ \pm}}} \sum_{k} \sqrt{q} \tilde{\pi}_{k}^{ \pm}\left(\tau_{ \pm}\right) e^{i k \xi_{ \pm}}$,

where complex-valued Fourier modes $\tilde{\phi}_{k}^{ \pm}$and $\tilde{\pi}_{k}^{ \pm}$are subject to the reality condition as we are considering the scalar field $\varphi$ to be a real-valued field. One may check that the Kronecker delta and the Dirac delta can now be expressed as

$$
\begin{aligned}
& \int d \xi_{ \pm} \sqrt{q} e^{i\left(k-k^{\prime}\right) \xi_{ \pm}}=V_{ \pm} \delta_{k, k^{\prime}} \\
& \sum_{k} e^{i k\left(\xi_{ \pm}-\xi_{ \pm}^{\prime}\right)}=V_{ \pm} \delta\left(\xi_{ \pm}-\xi_{ \pm}^{\prime}\right) / \sqrt{q} .
\end{aligned}
$$

The Eqs. (26) and (27) together allow the values of the wave-vector to be $k \in\left\{k_{l} \mid k_{l}=2 \pi l / L_{ \pm}\right\}$with $l$ being a non-zero integer. Using Fourier modes, the scalar field Hamiltonian (21) for both the observers can be expressed as $H_{\varphi}^{ \pm}=\sum_{k} N \mathcal{H}_{k}^{ \pm}$where the Hamiltonian density for the $k^{\text {th }}$ mode is

$\mathcal{H}_{k}^{ \pm}=\frac{1}{2} \tilde{\pi}_{k}^{ \pm} \tilde{\pi}_{-k}^{ \pm}+\frac{1}{2}|k|^{2} \tilde{\phi}_{k}^{ \pm} \tilde{\phi}_{-k}^{ \pm}$

The Poisson bracket between the Fourier modes and their conjugate momenta can be expressed as

$\left\{\tilde{\phi}_{k}^{ \pm}, \tilde{\pi}_{-k^{\prime}}^{ \pm}\right\}=\delta_{k, k^{\prime}}$.

\subsubsection{Relation between Fourier modes}

In order to establish the relation between the Fourier modes of two asymptotic observers, firstly we note that the matter field being scalar, it can be expressed in general as $\varphi\left(\tau_{-}\left(\tau_{+}, \xi_{+}\right), \xi_{-}\left(\tau_{+}, \xi_{+}\right)\right)=\varphi\left(\tau_{+}, \xi_{+}\right)$. Further, in the standard formulation of the Hawking effect, the observer near the $\mathscr{I}^{-}$, deals with the ingoing field modes for them $v=t+r_{\star}=\left(\tau_{-}-(\sqrt{2}-1) \xi_{-}\right) / \sqrt{2}$ is constant. On the other hand, the observer near $\mathscr{I}^{+}$deals with the outgoing field modes for them $u=t-r_{\star}=\left(\tau_{+}-(\sqrt{2}-1) \xi_{+}\right) / \sqrt{2}$ is constant. This aspect allows one to get a relation between the field momenta [38] as

$\Pi\left(\tau_{+}, \xi_{+}\right)=\left(\partial \xi_{-} / \partial \xi_{+}\right) \Pi\left(\tau_{-}, \xi_{-}\right)$.

The Fourier modes and the conjugate momenta on a given hyper-surface labeled by $\tau_{+}^{0}$, as seen by the observer $\mathbb{O}^{+}$, can be expressed using the modes corresponding to the observer $\mathbb{O}^{-}$, on a given hyper-surface labeled by $\tau_{-}^{0}$, as

$\tilde{\phi}_{\kappa}^{+}\left(\tau_{+}^{0}\right)=\sum_{k} \tilde{\phi}_{k}^{-}\left(\tau_{-}^{0}\right) F_{0}(k,-\kappa)$,

$\tilde{\pi}_{\kappa}^{+}\left(\tau_{+}^{0}\right)=\sum_{k} \tilde{\pi}_{k}^{-}\left(\tau_{-}^{0}\right) F_{1}(k,-\kappa)$,

where the coefficient functions $F_{m}(k, \kappa)$ are given by

$F_{m}(k, \kappa)=\frac{1}{\sqrt{V_{-} V_{+}}} \int d \xi_{+}\left(\frac{\partial \xi_{-}}{\partial \xi_{+}}\right)^{m} e^{i k \xi_{-}+i \kappa \xi_{+}}$,

with $m=0,1$. The coefficient functions $F_{m}(k, \kappa)$ play the similar role as the Bogoliubov coefficients. Using the expression (32), it can be shown that $F_{0}(k, \kappa)$ and $F_{1}(k, \kappa)$ are related as [40]

$F_{1}( \pm|k|, \kappa)=\mp \frac{\kappa}{|k|} F_{0}( \pm|k|, \kappa)$.

The coefficient function $F_{0}(k, \kappa)$ is formally divergent as the integrand is purely oscillatory. However, it can be evaluated by introducing a suitable regulator $\delta$ such that $\lim _{\delta \rightarrow 0} F_{0}^{\delta}( \pm|k|, \kappa)=F_{0}( \pm|k|, \kappa)$ and the regulated coefficient function can be evaluated as $[38,41]$

$F_{0}^{\delta}( \pm|k|, \kappa)=\frac{\left(2 r_{s}\right)^{-\beta}|k|^{-\beta-1}}{\sqrt{V_{-} V_{+}}} e^{ \pm i \pi(\beta+1) / 2} \Gamma(\beta+1)$,

where $\Gamma(\beta+1)$ is the Gamma function and $\beta=\left(2 i \kappa r_{s}+\delta-\right.$ 1). From the Eqn. (34), one can deduce an important relation as follows

$F_{0}^{\delta}(-|k|, \kappa)=e^{2 \pi r_{s} \kappa-i \delta \pi} F_{0}^{\delta}(|k|, \kappa)$.

\subsubsection{Number density of Hawking quanta}

Using the Eqs. (30), (31), (33) and (35) one can express the Hamiltonian density (28) corresponding to the positive frequency modes i.e. $\kappa>0$ for the observer $\mathbb{O}^{+}$in terms of the Fourier modes of the observer $\mathbb{O}^{-}$as [38]

$$
\frac{\mathcal{H}_{\kappa}^{+}}{\kappa}=\frac{h_{\kappa}^{1}}{\kappa}+\frac{e^{2 \pi \kappa / \varkappa}+1}{e^{2 \pi \kappa / \varkappa}-1}\left[\frac{1}{\zeta(1+2 \delta)} \sum_{l=1}^{\infty} \frac{1}{l^{1+2 \delta}} \frac{\mathcal{H}_{k_{l}}^{-}}{k_{l}}\right],
$$


where $\varkappa=1 /\left(2 r_{s}\right)$ is the surface gravity at the Schwarzschild event horizon and $\zeta(1+2 \delta)=\sum_{l=1}^{\infty} l^{-(1+2 \delta)}$ is the Riemann zeta function. The term $h_{\kappa}^{1}=\sum_{k \neq k^{\prime}}\left[\frac{1}{2} F_{1}(k,-\kappa) F_{1}\left(-k^{\prime}, \kappa\right)\right.$ $\left.\tilde{\pi}_{k}^{-} \tilde{\pi}_{-k^{\prime}}^{-}+\frac{1}{2}|\kappa|^{2} F_{0}(k,-\kappa) F_{0}\left(-k^{\prime}, \kappa\right) \tilde{\phi}_{k}^{-} \tilde{\phi}_{-k^{\prime}}^{-}\right]$being linear in Fourier modes and their conjugate momenta, would drop out from the vacuum expectation value. It is well known that the Fourier modes corresponding to a massless free scalar field can be viewed as a system of decoupled harmonic oscillators which can also be seen from the Eq. (28). Therefore, in Fock quantization $\left\langle\hat{\mathcal{H}}_{k}^{-}\right\rangle \equiv\left\langle 0_{-}\left|\hat{\mathcal{H}}_{k}^{-}\right| 0_{-}\right\rangle=\frac{1}{2}|k|$ where the state $\left|0_{-}\right\rangle$refers to the vacuum state of the observer $\mathbb{O}^{-}$. Consequently, the expectation value of the number density operator $\hat{N}_{\kappa}^{+} \equiv \hat{\mathcal{H}}_{\kappa}^{+} / \kappa-\frac{1}{2}$ corresponding to the observer $\mathbb{O}^{+}$, in the vacuum state of the observer $\mathbb{O}^{-}$can be evaluated as

$N_{\omega} \equiv\left\langle\hat{N}_{\omega=\kappa}^{+}\right\rangle=\frac{1}{e^{2 \pi \omega / \varkappa}-1}=\frac{1}{e^{\left(4 \pi r_{s}\right) \omega}-1}$.

The Eq. (37) corresponds to a thermal spectrum of bosons at the temperature $T_{H}=\varkappa /\left(2 \pi k_{B}\right)=1 /\left(4 \pi r_{s} k_{B}\right)$. This phenomenon is referred to as the Hawking effect and associated temperature is known as the Hawking temperature.

\section{Discussions}

In this article we have presented an exact analytical derivation of the Hawking effect in canonical formulation where one does not need to deal with the matter diffeomorphism generator. In order to achieve this simplification, we have introduced a new set of coordinates in which the resultant spacetime metric is diagonal. Consequently, the foliation of the spacetime into spatial hyper-surfaces, which is required for canonical derivation, does not introduce any shift vector. Therefore, these new coordinates lead to a much simpler canonical derivation of the Hawking effect compared to the one reported in Ref. [38] where one uses the so-called near-null coordinates. Clearly, these coordinates would be quite useful for testing various new quantization techniques $[39,41-46]$. We have mentioned earlier that the spacetime metric is diagonal in these new coordinates and up to a scaling the metric is similar to a conformally transformed Minkowski metric. However, it can be checked that these new coordinates cannot be obtained simply by applying a Lorentz boost from $\left(t, r_{\star}\right)$ coordinates. In this context we may mention that it would be quite interesting to use the canonical formulation as given here, to study the issue of ambiguity in the expression of Hawking temperature due to inequivalent choices of the inertial frames as shown by 't Hooft [47-53].

Acknowledgements We would like to thank Gopal Sardar, Subhajit Barman and Saumya Ghosh for many useful discussions. CS would like to thank IISER Kolkata for supporting this work through a doctoral fellowship.
Data Availability Statement This manuscript has no associated data or the data will not be deposited. [Authors' comment: The work is purely analytical. We have not utilized any particular external set of data to produce the results.]

Open Access This article is licensed under a Creative Commons Attribution 4.0 International License, which permits use, sharing, adaptation, distribution and reproduction in any medium or format, as long as you give appropriate credit to the original author(s) and the source, provide a link to the Creative Commons licence, and indicate if changes were made. The images or other third party material in this article are included in the article's Creative Commons licence, unless indicated otherwise in a credit line to the material. If material is not included in the article's Creative Commons licence and your intended use is not permitted by statutory regulation or exceeds the permitted use, you will need to obtain permission directly from the copyright holder. To view a copy of this licence, visit http://creativecomm ons.org/licenses/by/4.0/.

Funded by $\mathrm{SCOAP}^{3}$.

\section{References}

1. S.W. Hawking, Comm. Math. Phys. 43, 199 (1975)

2. S. Carroll, Spacetime and geometry. An introduction to general relativity (AW, 2004)

3. B.F. Schutz, A first course in general relativity (Cambridge University Press, Cambridge, 1985)

4. S.A. Fulling, Lond. Math. Soc. Student Texts 17, 1 (1989)

5. R. M. Wald, General relativity (University of Chicago Press, 1984), first edition ed

6. S. Singh, S. Chakraborty, Phys. Rev. D 90, 024011 (2014). arXiv: 1404.0684

7. T. Dray, G.T. Hooft, Commun. Math. Phys. 99, 613 (1985)

8. H. Kawai, Y. Matsuo, Y. Yokokura, Int. J. Mod. Phys. A 28, 1350050 (2013). arXiv: 1302.4733

9. L. Parker and D. Toms, Quantum field theory in curved spacetime: quantized fields and gravity, Cambridge monographs on mathematical physics (Cambridge University Press, Cambridge, 2009), 1 st ed

10. D. Singleton, S. Wilburn, Phys. Rev. Lett. 107, 081102 (2011). arXiv: 1102.5564

11. S. Bhattacharya, A. Lahiri, Eur. Phys. J. C 73, 2673 (2013). arXiv: 1301.4532

12. S. Singh, C. Ganguly, T. Padmanabhan, Phys. Rev. D 87, 104004 (2013). arXiv: 1302.7177

13. A.S. Lapedes, J. Math. Phys. 19, 2289 (1978)

14. P.-M. Ho, JHEP 08, 096 (2015). arXiv: 1505.02468

15. T. Jacobson, Lect. Notes Phys. 870, 1 (2013). arXiv:1212.6821

16. S.B. Giddings, W.M. Nelson, Phys. Rev. D 46, 2486 (1992)

17. P.-H. Lambert, PoS (Modave, 2013), arXiv: 1310.8312

18. K. Fredenhagen, R. Haag, Comm. Math. Phys. 127, 273 (1990)

19. T. Jacobson, in Lectures on quantum gravity. Proceedings, School of Quantum Gravity, Valdivia, Chile, 2002 (2003), pp. 39-89. arXiv:gr-qc/0308048

20. C. Kiefer, in DPG School of Physics (Course 2): Galactic Black Hole 2001 Bad Honnef, Germany, August 26-31, 2001 (2002). arXiv:astro-ph/0202032

21. J. H. Traschen, in Mathematical methods in physics. Proceedings, Winter School, Londrina, Brazil, August 17-26, 1999 (1999). arXiv:gr-qc/0010055

22. S. Chakraborty, S. Singh, T. Padmanabhan, JHEP 06, 192 (2015). arXiv: 1503.01774

23. S. Chakraborty, K. Lochan, Universe 3, 55 (2017). arXiv: 1702.07487 
24. S. Carlip, Int. J. Mod. Phys. D 23, 1430023 (2014). arXiv: 1410.1486

25. B.S. DeWitt, Phys. Rep. 19, 295 (1975)

26. L. H. Ford, in Particles and fields. in Proceedings, 9th Jorge Andre Swieca Summer School, Campos do Jordao, Brazil, 1997, pp. 345388. arXiv:gr-qc/9707062

27. S. Hollands, R.M. Wald, Phys. Rept. 574, 1 (2015). arXiv: 1401.2026

28. T. Padmanabhan, Rept. Prog. Phys. 73, 046901 (2010). arXiv:0911.5004

29. S. Fulling, S. Ruijsenaars, Phys. Rep. 152, 135 (1987)

30. K.J. Hinton, J. Phys. A Math. Gener. 16, 1937 (1983)

31. M.K. Parikh, F. Wilczek, Phys. Rev. Lett. 85, 5042 (2000). arXiv:hep-th/9907001

32. M. Visser, Int. J. Mod. Phys. D 12, 649 (2003). arXiv:hep-th/0106111

33. P.C.W. Davies, J. Phys. A 8, 609 (1975)

34. R.M. Wald, Commun. Math. Phys. 45, 9 (1975)

35. K. Melnikov, M. Weinstein (2001). arXiv:hep-th/0109201

36. K. Melnikov, M. Weinstein, Int. J. Mod. Phys. D 13, 1595 (2004). arXiv:hep-th/0205223

37. M. Weinstein, Nucl. Phys. Proc. Suppl. 108, 68 (2002). arXiv:gr-qc/0111027

38. S. Barman, G.M. Hossain, C. Singha, Phys. Rev. D 97, 025016 (2018). arXiv:1707.03614
39. G.M. Hossain, V. Husain, S.S. Seahra, Phys. Rev. D 82, 124032 (2010). arXiv:1007.5500

40. S. Barman, G. M. Hossain (2018). arXiv:1809.09430

41. G.M. Hossain, G. Sardar, Class. Quant. Grav. 33, 245016 (2016). arXiv: 1411.1935

42. A. Ashtekar, S. Fairhurst, J.L. Willis, Class. Quant. Grav. 20, 1031 (2003). arXiv:gr-qc/0207106

43. H. Halvorson, Stud. Hist. Philos. Sci. Part B Stud. Hist. Philos. Modern Phys. 35, 45 (2004)

44. G. M. Hossain, G. Sardar (2016). arXiv:1606.01663

45. G.M. Hossain, G. Sardar, Phys. Rev. D 92, 024018 (2015). arXiv:1504.07856

46. S. Barman, G. M. Hossain, C. Singha (2017). arXiv:1707.03605

47. G.T. Hooft, J. Geom. Phys. 1, 45 (1984)

48. G.T. Hooft, Nucl. Phys. B 256, 727 (1985)

49. E.T. Akhmedov, V. Akhmedova, D. Singleton, Phys. Lett. B 642 , 124 (2006). arXiv:hep-th/0608098

50. E.T. Akhmedov, T. Pilling, D. Singleton, Int. J. Mod. Phys. D 17, 2453 (2008). arXiv:0805.2653

51. V. Akhmedova, T. Pilling, A. de Gill, D. Singleton, Phys. Lett. B 673, 227 (2009). arXiv:0808.3413

52. B.D. Chowdhury, Pramana 70, 593 (2008). arXiv:hep-th/0605197

53. E.T. Akhmedov, V. Akhmedova, T. Pilling, D. Singleton, Int. J. Mod. Phys. A 22, 1705 (2007). arXiv:hep-th/0605137 